\title{
Incidência de fungos e qualidade fisiológica de sementes de pinhão manso (Jatropha curcas L.) após o armazenamento criogênico
}

\author{
Míriam Goldfarb ${ }^{1 *}$ \\ Maria Elita Martins Duarte ${ }^{1}$ \\ Mário Eduardo R. M. Cavalcanti Mata ${ }^{1}$ \\ Luciana Cordeiro do Nascimento ${ }^{2}$ \\ Noelma Miranda de Brito \\ Francisca Maria Souto ${ }^{2}$ \\ ${ }^{1}$ Unidade Acadêmica de Engenharia Agrícola, Universidade Federal de Campina Grande \\ CEP 58429-900, Campina Grande - PB, Brasil \\ ${ }^{2}$ Centro de Ciências Agrárias, Universidade Federal da Paraíba, Campus III, Areia - PB, Brasil \\ * Autor para correspondência \\ miriam.gold@hotmail.com
}

Submetido em 31/07/2009

Aceito para publicação em 15/12/2009

\section{Resumo}

O objetivo deste trabalho foi avaliar a incidência de fungos e qualidade fisiológica de sementes de pinhão manso crioarmazenadas a $-170^{\circ} \mathrm{C}$ e a $-196^{\circ} \mathrm{C}$. A pesquisa foi realizada nos setores de Criogenia/UFCG, Fitossanidade/UFPB e Embrapa Algodão. O material apresentava um teor de água de 8\% b.u., sendo 200 sementes armazenadas em botijões criogênicos com nitrogênio nas fases de vapor e líquido, com quatro períodos de armazenamento (0, 30, 60 e 90 dias) constituindo-se os tratamentos. Decorrido cada período, as sementes foram submetidas ao teste de sanidade (Blotter test) e germinação. Foi realizada a desinfestação superficial, e o material distribuído em placas de Petri sendo incubado a $25 \pm 2^{\circ} \mathrm{C}$ durante sete dias. A avaliação da incidência de fungos nas sementes foi realizada com microscópio estereoscópio através das observações das estruturas fúngicas, sendo os dados demonstrados em percentagem de sementes infectadas. O delineamento experimental foi inteiramente casualizado com temperaturas x dias de armazenamento. Aos 30 dias, foi detectada uma maior incidência fúngica, com predominância de Aspergillus sp., Cladosporium sp. e Fusarium sp. Concluiu-se que a crioconservação nas temperaturas criogênicas estudadas não reduziu a incidência de fungos nas sementes de pinhão manso e a qualidade fisiológica foi preservada durante a crioconservação.

Unitermos: análise sanitária e fisiológica, crioconservação, espécies oleaginosas

\section{Abstract}

Incidence of fungus and physiological quality of seeds of Jatropha curcas L. after cryogenic storage. The objective of this work was to evaluate the incidence of fungi in stored seeds of Jatropha curcas. The research 
was carried out at Cryogenic/UFCG, Sanity/UFPB and Cotton/Embrapa. The material for analysis showed an $8 \%$ water level, and 200 seeds were stored for treatment in cryogenic containers with nitrogen in the vapor and liquid phases. Four periods of crioconservation (0,30, 60 and 90 days), were employed. After each period, the seeds were tested for sanity (Blotter test) and germination. Superficial disinfestation, was carried out and seeds were distributed in Petri dishes, for incubation at $25 \pm 2^{\circ} \mathrm{C}$, over a period of 7 days. The evaluation of the incidence of fungi was carried out in a stereoscopic microscope with observation of fungal structures, and values were expressed as percentages of seeds with fungus. The statistical experiment was completely randomized with temperature $\mathrm{x}$ days of storage. Analysis of variance was conducted and the means were compared by Tukey's test at 5\%. After 30 days of cryogenic storage, a greater incidence of Aspergillus sp., Cladosporium sp. and Fusarium sp. was detected. It was concluded that crioconservation at cryogenic temperatures did not reduce the incidence of fungus on Jatropha curcas seeds. The physiological quality was preserved during the cryoconservation.

Key words: cryoconservation, oil species, sanity and physiological analyses

\section{Introdução}

O pinhão manso (Jatropha curcas L.) é uma espécie oleaginosa que, por suas potencialidades, vem sendo considerada economicamente importante e tem como característica principal resistência à seca, além da produção de óleo com todas as qualidades necessárias para ser transformado em óleo diesel (Arruda et al., 2004). Atualmente, o óleo extraído da semente tem sido utilizado para fins energéticos. A torta (co-produto da Jatropha) é tóxica, e, portanto, inadequada para alimentação animal; entretanto, tem potencial como adubo orgânico, pois o farelo residual apresenta elevados teores de nitrogênio, fósforo e potássio. Esta oleaginosa tem importância agrícola, por fornecer matéria-prima para a produção de biocombustível, isto é, devido ao seu teor de óleo que varia entre 30 e $40 \%$, com produção anual de 1.100 e $1.700 \mathrm{~L} / \mathrm{ha}$ (Nunes, 2007). Com essas características e por apresentar tolerância à seca, a cultura seria uma alternativa agrícola para a região Nordeste.

Jocker e Jepsen (2003) afirmam que as sementes de pinhão manso são ortodoxas e, à temperatura ambiente, podem permanecer viáveis por, pelo menos um ano; contudo, devido ao seu conteúdo de óleo não é recomendado um armazenamento prolongado uma vez que as sementes oleaginosas são mais suscetíveis ao ataque de patógenos e podendo ocorrer a rancificação dos ácidos graxos que compõem o óleo.

Severino et al. (2006) explicam que J. curcas é uma espécie que se encontra em processo de domesticação, e tem sido frequente a ocorrência de problemas relacionados às sementes que apresentam germinação irregular e perda do poder germinativo após alguns meses de armazenamento. Então é relevante investigar o efeito da crioconservação das sementes dessa espécie como uma alternativa ao armazenamento convencional, e prolongar os períodos de crioarmazenagem.

A técnica de crioconservação consiste em manter os produtos biológicos armazenados a temperaturas ultrabaixas, em geral a $-170^{\circ} \mathrm{C}$, na fase de vapor de nitrogênio, e a $-196^{\circ} \mathrm{C}$ na fase de nitrogênio líquido, condições em que o metabolismo do organismo biológico é inativado reduzindo os processos degradativos que resultam na deterioração do mesmo (Coelho, 2006). Com relação às sementes, este tipo de material pode ser crioconservado sem ocorrer alterações nas qualidades fisiológicas.

Segundo Aguiar et al. (2001), as sementes são eficientes meios de disseminação e de introdução de patógenos em áreas isentas. $\mathrm{O}$ inóculo inicial de epidemias pode depender da transmissão do patógeno pela semente, assim como reduzir a qualidade fisiológica das mesmas. Danos decorrentes da associação dos microorganismos patogênicos com sementes, não se limitam a perdas diretas da população em campo, mas envolvem outras implicações que podem provocar sérios danos em todo o sistema de produção (Machado, 1994; Marcos Filho, 2001). De acordo com Scott et al. (1982), grãos e sementes armazenados com teor de água acima de $13 \%$ podem apresentar desenvolvimento fúngico e o aquecimento da massa de grãos. Todos os fungos de armazenamento têm a capacidade de reduzir o poder germinativo da semente e causar a morte do embrião. Teores de água mais baixos na semente, próximos ao limite mínimo para o crescimento dos fungos, propiciam 
um ataque lento; todavia, na medida em que o teor de água da semente se eleva, ocorrem reduções no percentual germinativo em virtude do rápido crescimento de fungos (Popinigis, 1977).

Diante do exposto, o objetivo do trabalho foi avaliar a qualidade sanitária e fisiológica de sementes de pinhão manso armazenadas nas temperaturas criogênicas de $-170^{\circ} \mathrm{C}$, fase de vapor de nitrogênio e a $-196^{\circ} \mathrm{C}$, fase de nitrogênio líquido.

\section{Material e Métodos}

O presente estudo foi desenvolvido no Setor de Criogenia do Laboratório de Armazenamento e Processamento de Produtos Agrícolas da Unidade Acadêmica de Engenharia Agrícola da Universidade Federal de Campina Grande-PB, Laboratório de Fitopatologia, Departamento de Fitotecnia, da Universidade Federal da Paraíba, Campus II, Areia-PB e na Empresa Brasileira de Pesquisa AgropecuáriaEmprapa Algodão, Campina Grande-PB

Utilizaram-se sementes (material ainda não padronizado pelas Regras para Análise de Sementes) recém-colhidas de pinhão manso, safra 2007, provenientes da Embrapa Semi-Árido, em Petrolina, PE.

\section{Crioarmazenamento das sementes}

As sementes de pinhão manso, com teor de água em torno de $8 \%$ b.u. (Umidade de sementes em base úmida), umidade que apresentavam quando colhidas no campo, foram armazenadas em nitrogênio, a $-170^{\circ} \mathrm{C}$ na fase de vapor e $-196^{\circ} \mathrm{C}$ na fase líquida. Os períodos de armazenamento foram 0, 30, 60 e 90 dias. Para este procedimento, as sementes foram acondicionadas em tubos cilíndricos de alumínio (canister), separadas por dias de armazenamentos, e acondicionadas em botijões criogênicos isolados com vácuo parcial, conferindo ao mesmo capacidade de manter o nitrogênio em estado líquido $\left(-196^{\circ} \mathrm{C}\right)$ e no estado de vapor $\left(-170^{\circ} \mathrm{C}\right)$ (Cavalcanti Mata et al., 2004).

As sementes utilizadas como testemunha não foram submetidas ao crioarmazenamento, este tratamento foi descrito como sendo o período de zero dias.
Decorrido cada período, as sementes foram descongeladas pelo método de descongelamento gradativo sendo expostas as temperaturas de -196; $-170 ;-80 ; 10^{\circ} \mathrm{C}$ e temperatura ambiente com intervalo de três horas para cada temperatura, de acordo com metodologia proposta por Almeida et al. (2002). Com as sementes descongeladas, realizou-se o teste de sanidade, germinação e vigor.

\section{Teste de sanidade}

Utilizou-se o método de papel-filtro (blotter test), de acordo com metodologia proposta por Neergaard (1979) e Piña-Rodrigues (2004). Distribuíram-se 200 sementes em 20 placas de Petri (10 sementes por placa) para cada temperatura estudada. As sementes foram submetidas a uma desinfestação superficial em solução de álcool etílico a $70 \%$ por três minutos, hipoclorito de sódio a $1 \%$ por cinco minutos e duas lavagens em água destilada esterilizada (ADE) por um minuto, sendo dispostas sob papel-filtro esterilizado, em placas de Petri e incubadas a temperatura de $25 \pm 2^{\circ} \mathrm{C}$, durante sete dias, sob fotoperíodo de $12 \mathrm{~h}$. A avaliação da incidência dos fungos foi realizada através de microscópio estereoscópico e óptico, sendo os dados expressos em percentagem de sementes infectadas. O delineamento experimental foi inteiramente casualizado, com arranjo fatorial de duas temperaturas $\left(-170^{\circ} \mathrm{C}\right.$ e $\left.-196^{\circ} \mathrm{C}\right)$ e quatro períodos de armazenamento $(0,30,60$ e 90 dias $)$. A análise de variância e comparação das médias dos tratamentos foi realizada pelo teste de Tukey a 5\% de probabilidade, através do Assistat, versão 7.4 (Silva, 1996).

\section{Análise fisiológica de sementes}

Após crioconservadas e descongeladas, as sementes de pinhão manso foram submetidas ao teste de germinação e vigor. Como não existe, na literatura, um protocolo que descreva a metodologia para avaliar a qualidade fisiológica das sementes de pinhão manso, adotou-se nesta pesquisa, o teste de germinação (T.G) e não o teste padrão de germinação (T.P.G). Foram utilizadas quatro repetições de 50 sementes por tratamento. Para este teste, as sementes foram semeadas em bandejas de plástico com $56 \mathrm{~cm}$ de comprimento, $31,5 \mathrm{~cm}$ de largura e 
$7,0 \mathrm{~cm}$ de altura. O substrato utilizado foi a vermiculita de acordo com metodologia proposta por Piña-Rodrigues et al. (2004) adaptada das Regras para Análise de Sementes (Brasil, 1992). A vermiculita foi umedecida com água destilada e mantida úmida durante o teste.

Como o teste padrão para germinação de sementes de pinhão manso não foi descrito pelas Regras de Análise de Sementes (Brasil, 1992), consideraram-se germinadas as sementes que emitiram $30 \mathrm{~mm}$ de radícula no $14^{\circ}$ dia depois da semeadura. Este ensaio foi realizado em casa de vegetação com temperatura média de $24,1^{\circ} \mathrm{C}$ e umidade relativa de $63 \%$.

Os testes de vigor foram realizados pelo comprimento de plântula e peso de matéria seca, realizado no $14^{\circ}$ dia de avaliação. O peso seco foi calculado através da fórmula proposta por Vieira e Carvalho (1994).

O delineamento estatístico empregado foi o inteiramente casualizado, com arranjo fatorial de duas temperaturas $\left(-170\right.$ e $\left.-196^{\circ} \mathrm{C}\right)$ e quatro períodos de crioconservação ( $0,30,60$ e 90 dias), as médias foram comparadas pelo teste de Tukey a 5\% de probabilidade através do Assistat, versão 7.4 (Silva, 1996).

\section{Resultados e Discussão}

Observa-se, na Tabela 1, que a micoflora das sementes de pinhão manso submetidas ao armazenamento a temperaturas ultra-baixas é composta por fungos usualmente encontrados em grãos e sementes; entretanto, é importante ressaltar a presença de alguns gêneros, como Chalara sp. e Diplodia sp., os quais não são usualmente encontrados em sementes armazenadas.

O gênero Chalara sp. é importante agente causal de murchas vasculares, cancro e seca de ramos de árvores. No Brasil, ocorrem duas doenças causadas por este gênero, que são a seca da mangueira, muito comum em todas as regiões do país, e o cancro, que praticamente inviabilizou o cultivo desta árvore florestal no norte do país (Krugner e Bacchi, 1995).

Segundo Oliveira (2008), a espécie Chalara paradoxa é o agente causal da podridão negra do abacaxi, os sintomas principais são lesões escuras presente na superfície do fruto, caracterizando essa doença que é de pós-colheita. Assim, Chalara sp. é um patógeno que causa danos em produtos agrícolas durante a estocagem.

Sartori (2003) relata que o gênero Diplodia sp., são considerados agentes necrotróficos, apresentando fase parasitária na planta viva e fase saprofítica nos tecidos necrosados e restos culturais. Dessa maneira, o patógeno pode ser encontrado sobrevivendo como micélio no interior de sementes. O autor menciona que as espécies Diplodia maydis e Diplodia. macrospora sobrevivem em sementes de milho na forma de micélio, e as sementes infectadas constituem a principal forma de fonte de inóculo.

TABELA 1: Percentual de incidência de fungos em sementes de pinhão manso em função de dias de armazenamento nas temperaturas criogênicas de $-170^{\circ} \mathrm{C}$ na fase de vapor de nitrogênio e a- $196^{\circ} \mathrm{C}$ fase de nitrogênio líquido.

\begin{tabular}{|c|c|c|c|c|c|c|c|c|}
\hline \multirow{4}{*}{ Gêneros } & \multicolumn{8}{|c|}{ Dias } \\
\hline & \multicolumn{2}{|c|}{$\mathbf{0}$} & \multicolumn{2}{|c|}{30} & \multicolumn{2}{|r|}{60} & \multicolumn{2}{|c|}{90} \\
\hline & \multicolumn{8}{|c|}{ Temperaturas } \\
\hline & $-170^{\circ} \mathrm{C}$ & $-196^{\circ} \mathrm{C}$ & $-170^{\circ} \mathrm{C}$ & $-196^{\circ} \mathrm{C}$ & $-170^{\circ} \mathrm{C}$ & $-196^{\circ} \mathrm{C}$ & $-170^{\circ} \mathrm{C}$ & $-196^{\circ} \mathrm{C}$ \\
\hline Alternaria sp. & 2 & 2 & - & 1,5 & - & - & 2,7 & - \\
\hline Aspergillus sp. & - & - & 29,2 & 31,9 & 0,6 & 8,9 & 0,5 & 7,6 \\
\hline Chalara sp. & 0,5 & 0,5 & - & - & - & - & - & - \\
\hline Cladosporium sp. & - & - & 11,7 & 11,5 & 2,4 & 7,4 & 5,5 & - \\
\hline Curvularia sp. & - & - & - & 1,5 & - & - & - & - \\
\hline Colletrotrichum sp. & 3 & 3 & - & - & - & - & - & - \\
\hline Diplodia sp. & - & - & - & - & - & - & 8,3 & 2 \\
\hline Fusarium sp. & 14 & 14 & 7,4 & 7,3 & 4,8 & 3,7 & 5 & - \\
\hline Nigrospora sp. & 0,5 & 0,5 & - & - & - & - & - & - \\
\hline Rhizopus sp. & 1 & 1 & 1,5 & 4,7 & 1,8 & 6,7 & 5 & 1,3 \\
\hline Rhizoctonia sp. & 6 & 6 & - & - & - & 0,7 & 0,5 & - \\
\hline
\end{tabular}


Os fungos são os principais microrganismos que compõem a microflora das sementes em condições de armazenamento sendo os principais causadores de deteriorações e perdas durante este período (Puzzi, 2000).

De acordo com a Tabela 1, nos períodos de zero dias, ou seja, as sementes que não foram crioarmazenadas, sendo este o tratamento testemunha, os gêneros com maior percentual de incidência foram Fusarium sp, Rhizoctonia sp, Colletrotrichum sp e Alternaria sp.

Os gêneros de fungos mais encontrados foram, Aspergillus sp., Cladosporium sp. e Fusarium sp., dentre os quais, apenas Aspergillus sp. não foi identificado nas sementes não crioarmazenadas (Testemunha). Para os períodos de 30, 60 e 90 dias e para as temperaturas de $-170^{\circ} \mathrm{C}$ (vapor de nitrogênio) e $-196^{\circ} \mathrm{C}$ (nitrogênio líquido) esse gênero foi o predominante.

O gênero Aspergillus sp. é o mais comumente encontrado em grãos e sementes armazenados. Desenvolve-se em sementes e grãos cujo teor de água está em equilíbrio com umidades relativas entre $65-90 \%$, correspondendo a $13-14 \%$ b.u. Esses microorganismos se adaptam a ambientes com baixa umidade relativa e raramente atacam produtos com grau de umidade superior a $25 \%$ b.u. (Silva et al., 1995). Popinigis (1977) ressalta que as espécies dos gêneros Aspergillus sp. e Penicillium sp. se encontram entre os principais agentes deterioradores de sementes.

Os gêneros Cladosporium sp. e Fusarium sp. ocorrem sobre inúmeras espécies vegetais, especialmente como componente da microflora das sementes, ainda no campo e durante a estocagem armazenamento. Cladosporium sp. produz descoloração em grãos e sementes. Quando parasitam plantas, permanecem longos períodos no interior dos tecidos (Kimati et al., 1997). Fungos pertencentes ao gênero Fusarium sp. causam murcha e o amarelecimento das folhas de plantas cultivadas, sendo as espécies patogênicas transmitidas por sementes (Sallis et al., 2001; Farias, 2003). O gênero Rhizopus sp. foi detectado nas sementes crioarmazenadas e nas que não foram armazenadas; no entanto, apresentaram baixos percentuais de incidência. Tais microorganismos são causadores de podridões em frutos e vegetais armazenados e produzem mofo em diversos produtos agrícolas. É considerado um fungo com pouca importância econômica com relação às sementes; porém, sementes com elevada incidência desse patógeno devem ser desinfectadas (Barreto, 2001). Fassel e Barreto (2000) estudaram a qualidade fisiológica e sanitária de sementes de amendoim durante as etapas de beneficiamento e constataram maior incidência de Rhizopus sp., enquanto Lima et al. (1982) e Kabeere e Taligoola (1983) observaram que este organismo prejudica a germinação e o vigor além de causar tombamento de plântulas.

Os resultados da análise de variância para os períodos de armazenamento $\left(\mathrm{F}_{1}\right)$ indicam efeito significativo ao nível de $1 \%$ de probabilidade pelo teste F. Não houve efeito significativo para os fatores temperaturas de armazenamento $\left(\mathrm{F}_{2}\right)$ e a interação entre os fatores períodos de armazenamento $\left(\mathrm{F}_{2}\right)$ e temperaturas de armazenamento $\left(\mathrm{F}_{2}\right)$. O modelo linear e quadrático foram os que apresentaram efeito significativo. Houve efeito significativo apenas com relação aos períodos de armazenamento criogênico.

Observa-se na Tabela 2, que não houve diferença estatística com os valores médios de incidência de fungos com relação às temperaturas criogênicas em que as sementes foram expostas durante o armazenamento. Para os períodos de armazenamento criogênico, após 30 dias, foi detectado maior incidência de patógenos nas sementes com relação aos períodos de 0 (testemunha) 90 e 60 dias. Uma provável hipótese, é que os fungos já estavam presentes na superfície da semente, e alguns poderiam estar em estado latente. Dessa forma, durante o período de descongelamento gradativo, em que as sementes foram submetidas às temperaturas de $-80^{\circ} \mathrm{C}$, $10^{\circ} \mathrm{C}$ e temperatura ambiente, as mesmas tornaram-se um meio ideal para ocorrer quebra de dormência e assim promover a germinação dos esporos. Está hipótese foi ressaltada por Farias (2003) que crioconservou sementes de jatobá por sete dias e verificou uma maior incidência de fungos durante o período de crioconservação.

Zambenedetti et al. (2007), ao estudarem diferentes métodos de armazenamento de urediniósporos de Puccinia pachyrhizi, concluíram que o método que promoveu maior porcentagem de germinação de esporos 
foi após 60 dias de armazenamento em nitrogênio líquido. Campos et al. (2004) mencionaram que o método de conservação em nitrogênio líquido é considerado um dos métodos mais efetivos na preservação de fungos por períodos prolongados.

TABELA 2: Incidência de patógenos em sementes de pinhão manso em função dos dias de crioarmazenamento e temperaturas de $-170^{\circ} \mathrm{C}$ e a $-196^{\circ} \mathrm{C}$.

\begin{tabular}{lc}
\hline \multicolumn{1}{c}{ Fatores } & $\begin{array}{c}\text { Percentual de } \\
\text { incidência (\%) }\end{array}$ \\
\hline Temperatura criogênica $\mathbf{- 1 7 0}^{\circ} \mathbf{C}$ & $31,0 \mathrm{a}$ \\
Temperatura criogênica $\mathbf{- 1 9 6}^{\circ} \mathbf{C}$ & $31,2 \mathrm{a}$ \\
DMS & 8.87427 \\
Períodos de armazenamento (dias) & \\
$\mathbf{0}$ & $28,0 \mathrm{~b}$ \\
$\mathbf{3 0}$ & $60,5 \mathrm{a}$ \\
$\mathbf{6 0}$ & $17,4 \mathrm{~b}$ \\
$\mathbf{9 0}$ & $18,5 \mathrm{~b}$ \\
$\mathbf{D M S}$ & 16,76213 \\
$\mathbf{C V} \%$ & 39,09638 \\
\hline
\end{tabular}

Médias seguidas pela mesma letra não diferem significativamente pelo teste deTukey a $5 \%$ probabilidade.

Com relação ao armazenamento de sementes, Freitas et al. (2000) afirmam que as sementes ricas em óleo (25-40\%), exigem cuidados especiais durante o período de conservação, devido à degradação dos ácidos graxos, expressa pela rancificação do óleo, o que torna as sementes oleaginosas mais suscetíveis ao ataque de patógenos durante a estocagem; desta forma, o nível de deterioração em sementes armazenadas depende das condições ambientais durante esta fase. Marcos Filho (2005) menciona que os fungos patogênicos se desenvolvem em sementes com teores de água em equilíbrio com umidade relativa do ar superior a $68 \%$; neste contexto, o material biológico, quando armazenado às baixas temperaturas e umidades, pode ser conservado por períodos prolongados.

A Tabela 3 apresenta os resultados da análise estatística da germinação e do vigor das sementes de pinhão manso, nos períodos de crioarmazenamento em função das temperaturas. Observa-se que a média de germinação foi de $63,2 \%$; não houve diferença do ponto de vista estatístico entre os períodos de armazenamento e as temperaturas; acerca do vigor, notou-se diferença estatística entre o período zero (testemunha) em comparação com os períodos de armazenamento criogênico, sendo os valores que correspondem aos períodos de crioconservação estatisticamente superiores. Esses resultados são devidos, provavelmente, às mudanças de temperatura e à expansão do nitrogênio líquido durante o reaquecimento, que originaram quebra de dormência. Comportamento similar foi observado por Almeida et al. (2002) com a crioconservação de duas variedades de sementes de mamona; segundo os autores, para o fator período de crioconservação verifica-se maior

TABELA 3: Valores médios de germinação e vigor (comprimento de plântulas - C.P. e peso de matéria seca P.M.S) das sementes de pinhão manso em função das temperaturas e períodos de armazenamento.

\begin{tabular}{|c|c|c|c|}
\hline \multirow{2}{*}{ Fatores } & \multirow{2}{*}{ Germinação (\%) } & \multicolumn{2}{|c|}{ Vigor } \\
\hline & & C.P (cm) & P.M.S (g) \\
\hline $\begin{array}{l}\text { Temperatura criogênica } \\
-170^{\circ} \mathrm{C}\end{array}$ & $64 \mathrm{a}$ & $9,3 \mathrm{a}$ & $10,5 \mathrm{a}$ \\
\hline $\begin{array}{l}\text { Temperatura criogênica } \\
-196^{\circ} \mathrm{C}\end{array}$ & $62,3 \mathrm{a}$ & $9,5 \mathrm{a}$ & $10,6 \mathrm{a}$ \\
\hline DMS & 9,75610 & 0,85415 & 1,80207 \\
\hline \multicolumn{4}{|c|}{ Períodos de armazenamento (dias) } \\
\hline $\mathbf{0}$ & $60,4 \mathrm{a}$ & $7,7 \mathrm{~b}$ & $6,9 \mathrm{~b}$ \\
\hline 30 & $66,6 \mathrm{a}$ & $9,8 \mathrm{a}$ & $12,8 \mathrm{a}$ \\
\hline 60 & $63,3 \mathrm{a}$ & $10 \mathrm{a}$ & $11,4 \mathrm{a}$ \\
\hline 90 & $62,3 \mathrm{a}$ & $8,4 \mathrm{a}$ & $11,9 \mathrm{a}$ \\
\hline Média & 63,2 & 8,9 & 10,7 \\
\hline DMS & 18,42779 & 1,61336 & 3,40384 \\
\hline CV\% & 21,14745 & 12,40219 & 23,2749 \\
\hline
\end{tabular}

Médias seguidas pela mesma letra não diferem significativamente pelo teste de Tukey a $5 \%$ de probabilidade 
germinação aos 30 dias retornando ao nível inicial, aos 60 dias da crioconservação.

Constatou-se então, nesta pesquisa, que a crioconservação não reduziu a incidência de patógenos presentes nas sementes armazenadas. Nas temperaturas criogênicas, ocorreu a preservação da integridade do material biológico devido à redução das taxas metabólicas, sendo a micoflora presente também preservada. Os fungos de armazenamento observados com maior freqüência após a técnica de crioconservação, foram pertencentes aos gêneros Aspergillus sp., seguidos de Cladosporium sp. e Fusarium sp.

A germinação nas sementes de pinhão manso foi mantida durante os 90 dias de armazenamento nas temperaturas criogênicas de $-170^{\circ} \mathrm{C} \mathrm{e}-196^{\circ} \mathrm{C}$, então a crioconservação não alterou os índices germinativos.

Os índices de vigor foram maiores após os períodos de 30, 60 e 90 dias de crioarmazenagem.

As sementes mantiveram a viabilidade durante o armazenamento criogênico e os melhores resultados acerca do vigor foram obtidos nesse período.

\section{Agradecimentos}

A Coordenação de Aperfeiçoamento de Pessoal de Nível Superior (Capes) pela bolsa de pesquisa. A Empresa Brasileira de Pesquisa Agropecuária - Embrapa Algodão por fornecer matéria-prima e casa de vegetação. Ao Setor de Fitossanidade da Universidade Federal da Paraíba.

\section{Referências}

Aguiar, R. H.; Fantinatti, J. B.; Groth, D.; Usberti, R. 2001. Qualidade física, fisiológica e sanitária de sementes de girassol de diferentes tamanhos. Revista Brasileira de Sementes, 23 (1): 134139.

Almeida, F. de A. C.; Morais, A. M. de.; Carvalho, J. M. F. C.; Gouveia, J. P. G. 2002. Crioconservação de sementes de mamona das variedades nordestina e pernambucana. Revista Brasileira de Engenharia Agrícola e Ambiental, 6 (2): 295-302.

Arruda, F. P. de.; Beltrão, N. E. de M.; Andrade, A. P. de A.; Pereira, W. E.; Severino, L. S. 2004. Cultivo de pinhão manso (Jatropha curcas L.) como alternativa para o semi-árido nordestino. Revista Brasileira de Oleaginosas e Fibrosas, 8 (1):789-799.
Barreto, R. W. 2001. Fungos Fitopatogênicos. Universidade Federal de Viçosa,Viçosa, Brasil, 48pp.

Brasil. Ministério da Agricultura. 1992. Regras para análise de sementes. Brasília, Brasil: SNDA/DNDV/CLAV, 365pp.

Campos, A. K.; Mota, M. de.A.; Araújo, J. V.; Cecon, P. R. 2004. Atividade predatória, crescimento radial e esporulação de fungos predadores de nematóides Monacrosporium spp., submetidos à criopreservação. Ciência Rural, 34 (2): 465-469.

Cavalcanti Mata, M. E. R. M.; Rocha, M. do S.; Duarte, M. E. M. 2004. Teor de água limite para crioconservação de sementes de algodão arbóreo variedade 6M. Revista Brasileira de Produtos Agroindustriais, 6 (2): 179-189.

Coelho, R. R. P. 2006. Protocolo de crioconservação de sementes de algodão (Gossypium hirsutum L. raça Latifolium Hutch.) cultivares BRS 200 marrom e BRS verde. Tese de Doutorado, Universidade Federal da Paraíba, Brasil, 89pp.

Farias, D. C. de. 2003. Desenvolvimento de um protocolo para crioconservação de sementes de sementes de jatobá: Fitossanidade e cinética de congelamento. Dissertação de Mestrado, Universidade Federal de Campina Grande, Brasil, 86pp.

Fassel, S. A.; Barreto, M. 2000. Avaliação da qualidade fisiológica e sanitária de sementes de amendoim durante o beneficiamento. Revista Brasileira de Sementes, 22 (2): 126-130.

Freitas, R. A. de.; Dias, D. C. F. S.; Cecon, P. R.; Reis, M. S. 2000. Qualidade fisiológica e sanitária de sementes de algodão durante o armazenamento. Revista Brasileira de Sementes, 22 (2): 94-101.

Joker, D.; Jepsen, J. 2003. Jatropha curcas L. seed leaflet. Humleback, 83 (2): 1-2.

Kabeere, F.; Taligoola, H. K. 1983. Microflora and deterioration of soybean seeds in Uganda. Seed Science and Technology, 11 (2): 381-392.

Kimati, H.; Amorim, L.; Bergamin-Filho, A.; Camargo, L. E. A.; Rezende, J. A. M. 1997. Manual de fitopatologia. Doenças das plantas cultivadas. Ed Ceres, Piracicaba São Paulo, 398pp.

Krugner, T. L.; Bacchi, M. A. 1995. Fungos. In: Bergamin Filho, A. B.; Kimati, H. \& Amorim, L. (Eds). Manual de fitopatologia: Princípios e conceitos. Ed. Ceres, São Paulo, Brasil, p.80-83.

Lima, E. F.; Carvalho, L. P.; Carvalho, J. M. F. C. 1982. Comparação de métodos de análise sanitária e ocorrência de fungos em sementes de algodoeiro. Fitopatologia Brasileira, 7 (3): 401-406.

Machado, J. C. 1994. Podridões de tolerância de patógenos associados a sementes. Revisão Anual de Patologia de Plantas, 2: 229-263.

Marcos Filho, J. 2001. Pesquisa sobre vigor de sementes de hortaliças. Informativo ABRATES, 11: 63-75.

Marcos Filho, J. 2005. Fisiologia de sementes de plantas cultivas. Fealq, Piracicaba, Brasil, 495pp.

Neergaard, P. 1979. Seed pathology. The Macmillan, London, UK, 839pp.

Nunes, L. F. 2007. Caracterização de frutos, sementes e plântulas e cultivo de embriões de pinhão-manso (Jatropha curcas $\mathbf{L}$.). Dissertação de Mestrado, Universidade Federal de Lavras, Brasil, 78pp.

Oliveira, M. D de M. 2008. Controle pré e pós-colheita de doenças em abacaxizeiro. Dissertação de Mestrado, Universidade Federal da Paraíba, Brasil, 85pp. 
Piña-Rodrigues, F. C. M.; Figliolia, M. B.; Peixoto, M. C. 2004. Testes de qualidade. In: Ferreira, A. G. \& Borghetti, F. (Eds). Germinação do básico ao aplicado. Ed. Artmed, São Paulo, Brasil, p.53-65.

Popinigis, F. 1977. Fisiologia da semente. Ministério da Agricultura, AGIPAN, Brasília, Brasil, 289pp.

Puzzi, D. 2000. Abastecimento e armazenamento de grãos. Instituto Campineiro de Ensino Agrícola, Campinas, Brasil, 666pp.

Sallis, M. G. V.; Lucca-Filho, O. A.; Maia, M. de. S. 2001. Fungos associados às sementes de feijão-miúdo (Vigna unguiculata (L.) Walp.) produzidas no município de São José do Norte (RS). Revista Brasileira de Sementes, 23 (1): 36-39.

Sartori, F. A. 2003. Sementes de milho e restos culturais de aveia como fonte de inóculo para as podridões da base do colmo. Dissertação de Mestrado, Universidade de Passo Fundo, Brasil, 93pp.

Scott, M. L.; Nesheim, M. C.; Young, R. J. 1982. Nutrition of the chicken. $3^{\mathrm{a}}$ ed. Ed. Ithaca, New York, USA, 562p.
Severino, L. S.; Lima, R. de L. S.; Beltrão, N. E. de M. 2006. Germinação e crescimento inicial de plântulas de pinhão manso em função do peso da semente. Embrapa Algodão, Comunicado Técnico, 309: 3-4.

Silva, F. de A. S. E. 1996. The ASSISTAT Software: Statistical assistance. International Conference on Computers in Agriculture, Cancun, México, p.294-298.

Silva, J. de. S.; Donzeles, S. M. L.; Afonso, A. D. 1995. Qualidade dos grãos. In: Silva, J. de. S. (Ed.). Pré-processamento de produtos agrícolas. Instituto Maia, Niterói, Brasil, p.24-29.

Vieira, R. D.; Carvalho, N. M. 1994 Teste de vigor em sementes. Editora Afiliada, São Paulo, Brasil, 164pp.

Zambenedetti, E. B.; Alves, E.; Pozza, E. A.; Araújo, D. V. 2007. Germinação de urediniósporos de Phakopsora pachyrhizi em diferentes métodos de armazenamento. Summa Phytopathologica, 33 (1): 83-85. 